\title{
A Randomized Trial of Normothermic Preservation in Liver Transplantation
}

\author{
Abbreviations \\ AE Adverse event \\ ALT Alanine transaminase \\ AST Aspartate transaminase \\ ATP Adenosine triphosphate \\ BMI Body mass index \\ COPE Consortium for Organ Preservation in Europe \\ CVA Cerebrovascular accident \\ DBD Donation after brainstem death \\ DCD Donation after circulatory death \\ EAD Early allograft dysfunction \\ ET-DRI Eurotransplant donor risk index \\ fWIT Functional warm ischaemia time \\ GGT Gamma-glutamyl transferase \\ ITT Intention-to-treat \\ LDH Lactate dehydrogenase \\ MPI Minimal preservation injury \\ MRCP Magnetic resonance cholangiopancreatography \\ NAS Non-anastomotic stricture \\ NMP Normothermic machine perfusion \\ PNF Primary non-function \\ PP Per protocol \\ PRS Post-reperfusion syndrome \\ RRT Renal replacement therapy \\ SAE Serious adverse event \\ SCS Static cold storage \\ SPI Significant preservation injury \\ TPT Total preservation time
}

Corresponding authors:

- Professor Peter J Friend

Nuffield Department of Surgical Sciences, University of Oxford

Peter.friend@nds.ox.ac.uk

- Professor Constantin C Coussios

Institute of Biomedical Engineering, Department of Engineering Science, University of Oxford Constantin.coussios@eng.ox.ac.uk

- Dr David Nasralla

Nuffield Department of Surgical Sciences, University of Oxford

David.nasralla@nds.ox.ac.uk 
Author order and Institutions:

1. Dr David Nasralla

Nuffield Department of Surgical Sciences, University of Oxford, UK

2. Prof Constantin C Coussios

Institute of Biomedical Engineering, Department of Engineering Science, University of Oxford

3. Dr Hynek Mergental

Queen Elizabeth Hospital Birmingham, Birmingham, UK

4. Dr Mohammed Zeeshan Akhtar

a. Nuffield Department of Surgical Sciences, University of Oxford, UK

b. Target Discovery Institute, University of Oxford, UK

5. Dr Andrew Butler

University of Cambridge Department of Surgery, Addenbrooke's Hospital, Cambridge, UK

6. Dr Carlo Ceresa

Nuffield Department of Surgical Sciences, University of Oxford, UK

7. Ms Virginia Chiocchia

Centre for Statistics in Medicine and Surgical Intervention Trials Unit, Nuffield Department of Surgical Sciences, University of Oxford

8. Ms Susan J Dutton

Oxford Clinical Trials Research Unit, University of Oxford, UK

9. Prof Juan Carlos García-Valdecasas

Department of Hepatobiliopancreatic and Transplant Surgery, Hospital Clinic, Barcelona, Spain

10. Prof Nigel Heaton Institute of Liver Studies, King's College Hospital, London, UK

11. Dr Charles Imber

Department of Hepatopancreatobiliary and Liver Transplant Surgery, Royal Free Hospital, London, UK

12. Dr Wayel Jassem

Institute of Liver Studies, King's College Hospital, London, UK

13. Dr Ina Jochmans

a. Abdominal transplant surgery, Department of Surgery, University Hospitals Leuven, Leuven, Belgium

b. Lab of Abdominal Transplantation, Department of Microbiology and Immunology, KU Leuven, Leuven, Belgium

14. Prof John Karani

Institute of Liver Studies and Dept of Radiology, Kings College Hospital, London, UK

15. Dr Simon Knight

a. Nuffield Department of Surgical Sciences, University of Oxford, UK

b. Centre for Evidence in Transplantation, Clinical Effectiveness Unit, Royal College of Surgeons of England, UK

16. Dr Peri Kocabayoglu

Department of General, Visceral and Transplantation Surgery, University Hospital Essen, Germany

17. Prof Massimo Malagò

Department of Hepatopancreatobiliary and Liver Transplant Surgery, Royal Free Hospital, London, UK

18. Prof Darius Mirza

Queen Elizabeth Hospital Birmingham, Birmingham, UK

19. Prof Sir Peter Morris

a. Nuffield Department of Surgical Sciences, University of Oxford, UK 
b. Centre for Evidence in Transplantation, Clinical Effectiveness Unit, Royal College of Surgeons of England, UK

20. Dr Arvind Pallan

Department of Radiology, Queen Elizabeth Hospital Birmingham, Birmingham, UK

21. Prof Andreas Paul

Department of General, Visceral and Transplantation Surgery, University Hospital Essen, Germany

22. Dr Mihai Pavel

Department of Hepatobiliopancreatic and Transplant Surgery, Hospital Clinic, Barcelona, Spain

23. Dr Thamara Perera

Queen Elizabeth Hospital Birmingham, Birmingham, UK

24. Prof Jacques Pirenne

a. Abdominal transplant surgery, Department of Surgery, University Hospitals Leuven, Leuven, Belgium

b. Lab of Abdominal Transplantation, Department of Microbiology and Immunology, KU Leuven, Leuven, Belgium

25. Dr Reena Ravikumar

Nuffield Department of Surgical Sciences, University of Oxford, UK

26. Dr Leslie Russell

OrganOx Limited, Oxford, UK

27. Dr Sara Upponi

Department of Radiology, Addenbrooke's Hospital, Cambridge, UK

28. Prof Chris Watson

a. University of Cambridge Department of Surgery, Addenbrooke's Hospital, Cambridge, UK

b. National Institute of Health Research (NIHR) Cambridge Biomedical Research Centre, Cambridge, UK

29. Dr Annemarie Weissenbacher

Nuffield Department of Surgical Sciences, University of Oxford, UK

30. Prof Rutger Ploeg

Nuffield Department of Surgical Sciences, University of Oxford, UK

31. Prof Peter Friend

Nuffield Department of Surgical Sciences, University of Oxford, UK

Funding:

This study was funded by a European Commission Seventh Framework Programme (FP7) Grant (No 305934)

Competing Financial Interest Disclosure:

Professor Peter Friend is a co-founder, Chief Medical Officer and Consultant to OrganOx Limited and also holds shares in the company. Professor Constantin Coussios is a co-founder, Chief Technical Officer and Consultant to OrganOx Limited and also holds shares in the company. Neither Professor Friend nor Professor Coussios were involved in the selection, recruitment or transplantation of patients in this study.

\section{Summary}

Liver transplantation is a highly successful treatment, but is severely rationed by the shortage of donor organs. However, many potential donor organs cannot be used; this is because sub-optimal livers do not tolerate conventional cold storage and there is no reliable way to assess organ viability preoperatively. 
Normothermic machine perfusion maintains the liver in a physiological state, avoids cooling and allows recovery and functional testing. In a randomised trial enrolling 270 livers, we have shown that, when compared with conventional static cold storage, normothermic preservation is associated with a $50 \%$ lower level of graft injury, measured by hepatocellular enzyme release, despite a $50 \%$ lower rate of organ discard and a $54 \%$ longer mean preservation time. There was no significant difference in bile duct complications, graft or patient survival. If translated to clinical practice, these results would have a major impact on liver transplant outcomes and waiting list mortality.

\section{Introduction}

Liver transplantation is the accepted treatment for end-stage liver failure, with one and five year survivals in excess of $90 \%$ and $70 \%$ respectively [1]. With increasing rates of liver disease [2], the supply of transplantable organs is no longer able to meet demand. Paradoxically, despite substantial waiting list mortality (eg $21 \%$ in the UK), only $63 \%$ of UK deceased donor livers are transplanted [1]. Increasing numbers of deceased organ donors in many countries have not been matched by a corresponding rise in the number of transplantable organs. This is mainly because these additional donors tend to be high-risk - either declared dead by cardiovascular criteria (DCD), as opposed to brainstem death donors (DBD), or elderly with multiple co-morbidities (extended criteria donors). Such organs pose a greater risk to the recipient, with a higher probability that the liver will never function (primary non-function, PNF) or that it will lead to later complications, particularly biliary stricturing.

Despite many advances in liver transplantation, the method of organ preservation has changed very little in almost 30 years [3]. The liver is flushed and cooled with specialist preservation fluid, then stored in an ice-box. This process, static cold storage (SCS), has several limitations. Although SCS slows metabolism by 10 to 12 fold, significant anaerobic activity continues even at ice temperature [4]. This leads to ATP depletion and accumulation of succinate and other metabolites. These lead to the generation of reactive oxygen species [5] that are the basis of ischaemia-reperfusion injury, when the organ is re-exposed to oxygenated blood at the time of transplantation. This damage, exacerbated by any prior injury, limits the maximum safe preservation time of the donor organ. Once cooled, the cessation of normal cellular activity also makes functional assessment impossible.

These shortcomings are particularly problematic in the higher-risk donor organs that form an increasing proportion of today's liver transplant practice. The very severe ischaemia-reperfusion-related morbidity that characterises transplantation of such organs is now a major limitation in meeting the demand for lifesaving transplants. To combat the limitations imposed by cold storage a step change in preservation technology is required. In recent years, interest has developed in perfusion at physiological temperature (normothermic machine perfusion; NMP) [6-9].

During normothermic machine perfusion (NMP) the liver is perfused with oxygenated blood, medications and nutrients at normal body temperature to maintain a physiological milieu. Evidence from animal models of both DBD and DCD liver transplantation $[10,11]$ suggests that this improves the post-transplant survival of transplanted livers, and potentially enables the assessment of organ viability during preservation. The mechanism underlying these improved outcomes is at least partly related to the metabolic resuscitation of the organ that occurs with preservation under physiological conditions. This has been demonstrated through the replenishment of ATP levels [11] which in turn contributes to a reduction in the severity of ischaemia-reperfusion injury experienced after transplantation $[5,10]$.

There is increasing interest in the clinical application of NMP, with several cases described in the recent literature [6, 7]. In 2013 a phase I study by our group [9], demonstrated the safety and feasibility of NMP in 20 liver transplant recipients. . 
The present study is the first, randomised controlled trial to test the efficacy of machine perfusion against conventional cold storage. Livers from adult DBD or DCD donors were eligible for enrolment. Adults patients awaiting a liver only transplant, excluding those with fulminant liver failure, were eligible. If a suitable liver was allocated to a consented recipient, then the liver was randomised to either conventional SCS or NMP. In the SCS arm the organ retrieval, storage and the transplant were conducted according to standard practice. In the NMP arm, following removal from the donor, the liver was attached to the OrganOx metra ${ }^{\circledR}$ NMP device, where it was perfused throughout the duration of preservation (Figure 1), until the transplanting surgeon was ready to implant it, at which point it was removed from the device. The remainder of the recipient's care followed standard practice.

Daily during the first post-operative week, and at day 10 , day 30 , month six and month 12 , biochemical results were recorded as well as graft and patient survival data. At six months an MRI scan of the biliary tree (MRCP) was performed to assess evidence of biliary injury. Biological samples were collected and stored in a biobank from each liver and recipient enrolled in the study, for use in further mechanistic studies.

The primary endpoint was defined as the difference between the two treatment arms in the peak level of serum aspartate transaminase (AST) within seven days post-transplant. This hepatocellular enzyme is a clinically accepted biomarker, predictive of graft and patient survival [12].

\section{Results}

\section{Recruitment}

Between $26^{\text {th }}$ June 2014 and $8^{\text {th }}$ March 2016, 334 livers were randomised with 64 subsequently excluded (Figure 2). Following organ retrieval, a marked differential discard rate between the two trial arms resulted in 100 SCS and 120 NMP livers available for primary outcome reporting, with 101 SCS and 121 NMP available for secondary outcome analysis. This discrepancy in group size reduced the study power to $89.7 \%$.

One NMP liver was cold stored due to an accessory left hepatic artery arising from the aorta preventing effective cannulation. Eight NMP livers received machine perfusion for less than four hours (for logistic rather than technical reasons). All of these organs are included in the NMP arm as part of the modified ITT analysis. For the per protocol sensitivity analysis the eight livers perfused for less than four hours were excluded and the single NMP liver that was preserved using SCS was reassigned to the SCS group.

\section{Donor, Preservation and Recipient Characteristics (Table 1 and 2)}

NMP and SCS donor and recipient groups were well matched. The discard rate was higher in the SCS arm (24.1\%; 32/133) than the NMP arm (11.7\%; 16/137) (Extended Data Table 1). This difference was statistically significant (-12.4\% (95\% C.I. $-21.4 \%,-3.3 \%) ; p=0.008)$. One NMP discard was the result of a device malfunction in an already marginal organ (hepatic artery hypoperfusion due to pinch valve miscalibration; see Supplementary Information: Device Error Serious Adverse Event).

Functional warm ischaemia time (fWIT) applies only to DCD livers and was measured as the time from the onset of donor hypoxia (oxygen saturation $<70 \%$ ) or hypoperfusion (systolic blood pressure $<50 \mathrm{mmHg}$ ) until the start of cold aortic perfusion in the donor. The median fWIT was longer for NMP than SCS livers ( $21 \mathrm{~min}$ vs $16 \mathrm{~min} ; \mathrm{p}=0.003$ ).

Total preservation time (TPT) was measured from the start of cold aortic perfusion in the donor until graft reperfusion in the recipient. The median TPT was longer for NMP than SCS livers (11hr $54 \mathrm{~min}$ vs $7 \mathrm{hr} 45 \mathrm{~min}$; 
$p<0.001)$. Within the NMP arm, there was no significant difference in median perfusion time between DBD and DCD livers (9hr $55 \mathrm{~min}$ DBD vs $8 \mathrm{hr} 45 \mathrm{~min}$ DCD; $\mathrm{p}=0.449$ ).

Post-reperfusion haemodynamics were documented in 218 cases: post-reperfusion syndrome (PRS) was more common in the SCS (32/97) than the NMP group (15/121), a statistically significant difference ($20.6 \%, 95 \%$ C.I. $-31.6 \%$ to $-9.5 \%$; $p<0.001$ ). This was despite reduced requirement for vasopressors in NMP livers in the post-reperfusion period (Extended Data Tables 2a-c).

\section{Peak AST (primary outcome)}

Peak AST during the first 7 days post-transplant was reduced by $49.4 \%$ in the NMP group compared to SCS when adjusted by centre and donor type (geometric mean ratio $0.506,95 \%$ C.I. 0.388 to $0.659 p<0.001$ ). Unadjusted analysis (t-test) and sensitivity analysis undertaken in the per-protocol population confirmed these results.

Subgroup analysis showed that the effect of NMP was different in the two donor types (test for interaction $\mathrm{p}=0.012$ ), although it was statistically significant in both subgroups, with the reduction in peak AST being greater in DCD (73.3\% (95\% C.I. 53.7\% to 84.6\%, $p<0.001)$ ) than in DBD livers $(40.2 \%$ (95\% C.I. $19.3 \%$ to $55.7 \%, p=0.001)$ ). Subgroup analyses for MELD score and DRI showed no statistically significant differences (not reported). See extended data Table 3a-b and extended data Figure 1 for further analysis. See Table 3 for full Outcome results.

\section{Early Allograft Dysfunction (EAD)}

Data to assess EAD were available in 216 recipients: the odds of developing EAD in the NMP arm (12/119) were $74 \%$ less than the SCS (29/97) arm (odds ratio 0.263 (95\% C.I. 0.126, 0.550); $p<0.001$ ). A logistic regression model adjusted for donor type, MELD score and ET-DRI showed the adjusted odds of EAD in the NMP arm are approximately $72 \%$ less than in the cold storage arm (adjusted OR $0.276,95 \%$ C.I. 0.124 to $0.611 ; p=0.002$ ). The difference in EAD rates was partly a result of the difference in peak AST (described above), but also a reflection of differences in bilirubin. The mean bilirubin level in the first week postoperatively was lower in NMP recipients $(2.25 \mathrm{mg} / \mathrm{dL}(95 \%$ C.I. $1.23-4.28)$ ) than in the SCS group $(2.87 \mathrm{mg} / \mathrm{dL}(95 \%$ C.I. $1.52-5.00))(\mathrm{p}=0.029)$.

\section{Biliary Strictures on MRCP}

An MRCP was performed on 155 (81 NMP, 74 SCS) of the 222 transplanted trial patients. There was no significant difference in the rate of non-anastomotic strictures for DBD (NMP 7.4\% (4/54) vs SCS 5.4\% (3/55); $p=0.678$ ) or DCD (NMP 11.1\% (3/27) vs SCS 26.3\% (5/19); $p=0.180$ ) livers. Only one patient in each trial arm developed clinically relevant evidence of ischaemic cholangiopathy in the first year posttransplant, both of whom were re-transplanted.

Similarly, there was no significant difference in the rate of anastomotic strictures for DBD (NMP 40.7\% (22/54) vs SCS 41.8\% (23/55); $p=0.909)$ or DCD (NMP 48.1\% (13/27) vs SCS 57.9\% (11/19); $p=0.515)$ livers.

\section{Hospital Stay, Graft and Patient Survival}

There was no difference in median ITU stay ( 4 days NMP vs 4 days SCS; $p=0.339$ ), hospital stay (15 days NMP vs 15 days SCS; $p=0.926$ ) or the need for renal replacement therapy in the first post-operative week (2.7\% (95\% C.I. $-7.9 \%, 13.2 \%) ; p=0.621$ ). 
One NMP liver developed PNF (see Supplementary Information: Primary Non-Function Serious Adverse Event). There were no PNF cases in the SCS arm. Overall 10 recipients died during follow-up producing a one-year survival of 0.949 ( $95 \%$ C.I 0.890 to 0.977 ) in the NMP group and 0.958 (95\% C.I 0.902 to 0.982 ) in the SCS group ( $p=0.901$ ). Two deaths in the SCS group and three deaths in the NMP group were due to graft failure.

Graft survival at one year was 0.950 (95\% C.I 0.893 to 0.977 ) and 0.960 (95\% C.I 0.897 to 0.985 ) in the NMP and SCS groups respectively $(p=0.695)$. The causes of graft failure in the SCS arm were: hepatic artery thrombosis $(n=3)$; ischaemic cholangiopathy $(n=1)$. The causes of graft failure in the NMP arm were: hepatic artery thrombosis $(n=2)$; ischaemic cholangiopathy $(n=1)$; non-thrombotic infarction $(n=1)$; IVC occlusion ( $n=1)$; PNF $(n=1)$. (Extended Data Figure 2a-b).

For more detailed analysis of trial outcomes please see the Supplementary Information: Final Statistical Report.

\section{Machine Perfusion Characteristics Potentially Indicative of Organ Quality (Extended data}

\section{Fig 3a-d, Fig 4 and table 4a-b)}

The mean and standard deviations of all continuously monitored haemodynamic (arterial and portal flow), metabolic ( $\mathrm{pH}$ and lacate clearance), and synthetic (bile production) parameters by the third hour of NMP for all livers that went on to be successfully transplanted were:

- Hepatic artery flow: $280 \pm 120 \mathrm{ml} / \mathrm{min}$

- Portal vein flow: $1.11 \pm 0.2 \mathrm{~L} / \mathrm{min}$

- $\mathrm{pH}$ : $7.31 \pm 0.17$

- Lactate clearance: From $9.99 \pm 3.13 \mathrm{mmol} / \mathrm{L}$ at 15 minutes NMP to $0.93 \pm 0.63 \mathrm{mmol} / \mathrm{L}$ by 4 hours NMP

- Bile production: $\quad 9.17 \pm 11.16 \mathrm{ml} / \mathrm{hr}$. Notably, 18 transplanted NMP livers produced no/minimal bile during perfusion. All but one of these functioned after transplant. There was no correlation with post-transplant liver function or later development of non-anastomotic biliary strictures.

One NMP liver developed PNF. This liver was persistently acidotic with lactate $>4 \mathrm{mmol}$ for the duration of NMP. No other liver with these characteristics was transplanted.

Following transplant, 28 livers displayed minimal preservation injury (MPI; peak AST < $250 \mathrm{IU} /$ litre) and 25 showed evidence of severe preservation injury (SPI; peak AST > $1000 \mathrm{IU} /$ litre). The donors in these groups were well matched in all characteristics, other than sex. During NMP there was a difference in baseline perfusate ALT (MPI 171 IU/L vs SPI 669 IU/L; p=0.005) and LDH (MPI 1073 IU/L vs SPI 1838 IU/L; p=0.01) between the two groups. Levels of these enzymes, as well as GGT, increased more rapidly during the first 8 hours of NMP in the SPI group (ALT +56 IU/L vs +461 IU/L, $p<0.001 ; \mathrm{LDH}+483 \mathrm{IU} / \mathrm{L}$ vs +980 IU/L, $p=0.06$; $\mathrm{GGT}+23 \mathrm{IU} / \mathrm{L}$ vs $+104 \mathrm{IU} / \mathrm{L}, \mathrm{p}=0.004)$. MPI livers showed a reduction in measurable levels of haemolysis (haemolysis index) as NMP progressed, in contrast to SPI livers where this rose (MPI $-0.04 \mathrm{U}$ vs SPI +0.09 $\mathrm{U} ; \mathrm{p}=0.03$ ). Bile production was greater in the MPI group (MPI $13.1 \mathrm{ml} / \mathrm{hr}$ vs SPI $7.8 \mathrm{ml} / \mathrm{hr} ; \mathrm{p}=0.03$ ). Lactate clearance was similar in each group. Post-reperfusion syndrome was less common in the MPI group (MPI $0 \%(0 / 28)$ vs SPI $24 \%$ (6/25); $p=0.007)$. One NMP liver with perfusate transaminases in excess of 20,000 IU/L was transplanted successfully.

\section{Adverse Events (Extended data table 5a-c and table 6)}

The proportion of patients for whom adverse events were reported was similar in the two arms (55.4\% NMP (95\% C.I. $46.1 \%$ to $64.4 \%)$ vs $57.4 \%$ SCS (95\% C.I. $47.2 \%$ to $67.2 \%)$ ) with a larger total number of 
events reported for SCS livers (128 NMP vs 164 SCS). Of these, a greater proportion of the Serious Adverse Events (Clavien-Dindo grade $\geq \mathrm{IIlb}$ ) were in the SCS than NMP arm (16.4\% NMP (21/128) vs $22 \%$ SCS (36/164)). No statistical tests were applied to these data.

\section{Discussion}

This is the first randomised controlled trial to compare any type of machine perfusion technology with conventional static cold storage preservation in human liver transplantation.

The trial demonstrated significant reductions in peak AST and EAD rates in NMP livers; this is of clinical relevance as both are clinically accepted biomarkers for long-term graft and patient survival $[12,13]$. These benefits are consistent with previous animal work [10] and the Phase I clinical study [9] that preceded this trial, both of which showed post-transplant AST reductions in NMP livers. No differences were seen in graft or patient survival: a much larger trial would be required to test this outcome. It is notable that these reductions in peak AST and EAD rates were achieved in the context of improved organ utilization and longer preservation times, both of which have implications in terms of addressing the donor shortage and logistical barriers that currently limit liver transplants.

DCD donors represent a largely untapped source of organs, comprising $42 \%$ of UK deceased donors, but only $21 \%$ of transplanted livers [14]. Utilisation of DCD livers is limited by poorer outcomes (primary nonfunction and ischaemic cholangiopathy) compared with DBD livers. Allowing the limitations of small group analyses, in this study NMP DCD liver primary outcome data were superior to those of both DCD and DBD livers preserved using SCS. In fact, the primary outcome of DCD NMP livers was superior to that of DBD livers preserved by NMP: this was possibly due to a selection bias, both of donors (lower threshold to decline DCD donors) and recipients (fitter patients selected for higher-risk organs). The AST differences are in the context of longer functional warm ischaemia times, longer preservation times and fewer organ discards in the NMP arm, suggesting that NMP may be achieving the desired objective of increasing organ utilisation without compromising outcome. If these findings were translated into clinical practice, the increase in organ utilisation would have substantial implications for waiting list mortality, which is currently approximately one in five patients [1].

The longer preservation times in the NMP group were not planned, but were all within the maximum perfusion time defined in the protocol. There was no stipulation in the trial protocol that the preservation times should be matched. As clinicians gained experience, it appeared that some centres had started to organise their operating schedule according to the preservation method, although no overall difference between arms was seen in the proportion of transplants occurring in daylight hours. If, as appears to be the case, NMP can safely extend preservation times without compromising outcomes, this will have implications for operating department planning as well as organ utilisation.

There were over $50 \%$ fewer discarded organs in the NMP group, resulting in $20 \%$ more transplanted livers (121 NMP vs 101 SCS). The SCS discard rate of $23.7 \%$ was higher than the $17 \%$ reported in UK registry data [14], and may reflect the high proportion of DCD livers enrolled in the trial; the discard rate of retrieved DCD livers in the UK is 30\% [14]. This reported difference in organ utilisation is likely to be an underestimate of the full potential impact that NMP could have on transplant numbers. The trial stipulated that only livers considered transplantable according to standard practice could be enrolled. For the full extent of improved organ utilisation to be measured, livers would need to be randomised to NMP or SCS prior to being offered for transplant; this should form the basis of a future study. An increase of $20 \%$ or more in the number of transplantable donor livers would have a transformative effect on the mortality on liver transplant waiting lists around the world. 
The haemodynamic characteristics of the NMP recipients following reperfusion were measurably superior to those of SCS recipients, in line with previously reported findings [15]. This did not translate into a difference in ITU stay, hospital stay or need for renal replacement therapy (RRT) between the two groups, despite previous reports showing a correlation between peak-AST and RRT [16]. The magnitude of the reperfusion syndrome is a factor in determining the eligibility of the sickest patients for high-risk organs, due to the limited capacity of such patients to tolerate cardiovascular instability; NMP might therefore increase the options for the most urgent patients.

Perhaps the greatest limitation to more widespread utilisation of DCD livers is the high rate of clinicallysignificant non-anastomotic biliary strictures (NAS) which lead to a high rate of graft failure; this is believed to develop due to the vulnerability of the biliary tree to prolonged warm ischaemia. The rate of NAS in the NMP DCD group (11.1\%) was lower than in SCS (26.3\%) livers, despite longer functional warm ischaemia times. This did not reach statistical significance which may be a function of sample size; the trial was not powered for this outcome. Reported rates of NAS in DCD transplants vary from $10 \%$ to $30 \%[17,18]$ but these are in patients with symptoms suggesting biliary pathology, rather than those only apparent on imaging; biliary investigations are usually only performed for clinical indications (typically deranged liver function). Prior to this study, the radiological incidence of both anastomotic and non-anastomotic strictures in asymptomatic patients was unknown; in particular, there is no real benchmark against which to compare the rate of NAS seen in the DCD SCS group. Apart from the two patients retransplanted for ischaemic cholangiopathy, almost all of the remaining patients with radiological evidence of NAS had normal liver function at one year; this questions the clinical relevance of a protocol MRCP at six months. The longer-term follow-up of these patients will shed light on the importance of a radiological diagnosis of biliary stricturing in patients with normal graft function, and the role of MRCP as an endpoint in future trials.

As well as demonstrating improved graft preservation, this trial tested the feasibility, usability and safety of NMP, a vital component of the evaluation of any new technology. It showed that the logistical challenges of NMP can be met successfully within clinical practice. Over 120 NMP livers were transplanted in seven transplant centres across four European countries. Nonetheless, adoption of this technology into clinical practice may necessitate changes in the organ retrieval process, particularly with respect to technical support and transport arrangements. It remains to be seen whether NMP is required for the full duration of an organ's preservation or can equally well be applied after a short period of SCS when the organ reaches the transplanting centre - this would simplify the logistics but may not be suitable for the most marginal organs [19]. A Phase 2 study to test this has recently completed enrolment in the UK (NCT03176433).

For this new technology to be supported by healthcare funders, a health-economic case is needed. The results of this study suggest that benefits will accrue not only from improved early graft function and transplantation logistics, but also from improved utilisation. Secondary economic benefits will accrue from logistic changes, enabling transplants to be moved predominantly into daytime operating, with reduction in staffing costs and likely improvements in outcome. More timely intervention will also bring economic benefits - earlier transplantation is associated with lower morbidity and cost.

The effects of NMP demonstrated in this study are unequivocal with respect to the primary endpoint, implying a benefit in livers currently used for transplantation. However, the greatest benefit may be realised by applying this technology to livers outside current acceptance criteria, in order to transplant organs currently deemed untransplantable. Algorithms to assess organ viability, based on data obtained during NMP, will be essential if this potential is to be realised [10]. This study sheds some light on which perfusion parameters may be used to assess organ quality: bile production, acid-base stability, lactate clearance, perfusate transaminase levels, falling measurable haemolysis - all correlate with the degree of 
preservation injury evident after transplantation. However, all but one of the livers transplanted in the NMP group functioned postoperatively, including one NMP liver with perfusate transaminases in excess of 20,000 IU/L and 18 livers with minimal bile production. Data from much larger numbers of NMP transplants (typically from a registry) would be required to determine specific markers of viability.

The significance of bile production during NMP is unclear. Preliminary evidence from our group [20] suggests that preservation injury causes impaired hepatocellular uptake of bile salts. We have shown evidence of progressive accumulation of bile salts in the perfusate of livers with high post-transplant transaminase levels; something which also correlates with poor bile production during NMP. The extent and nature of the injury required to produce this effect is not clear but does appear to reflect organ quality rather than viability.

High-risk organs (e.g. those with steatosis) may benefit from therapeutic interventions delivered during NMP: several groups are exploring potential strategies, including stem cell treatments, de-fatting agents and immunological modification of the organ. Future trials may be needed to test formally the size of the effect of NMP on organ utilisation; for this it will be necessary to randomise livers at the time of organ offering rather than the time of retrieval. Organ utilisation, or organ utilisation with 12-month graft survival ('functional utilisation') would be a logical primary endpoint for a study of this sort.

This study is the first formal clinical evaluation of a novel technology in liver transplantation, and could herald the start of a new era of intervention during organ preservation. It represents the first, necessary step in demonstrating that NMP is feasible, safe and effective in clinical practice; the fact that the study has definitively met its primary endpoint should now open the door to explore this technology's wider potential. 
1. NHS Blood and Transplant, Annual Report on Liver Transplantation 2016/2017. September 2017.

2. APPHG, The All-Party Parliamentary Hepatology Group (APPHG) Inquiry into Improving Outcomes in Liver Disease. 2014.

3. Todo, S., et al., Extended preservation of human liver grafts with UW solution. JAMA, 1989. 261(5): p. 711-4.

4. Clavien, P.A., P.R. Harvey, and S.M. Strasberg, Preservation and reperfusion injuries in liver allografts. An overview and synthesis of current studies. Transplantation, 1992. 53(5): p. 957-78.

5. Chouchani, E.T., et al., Ischaemic accumulation of succinate controls reperfusion injury through mitochondrial ROS. Nature, 2014. 515(7527): p. 431-435.

6. Watson, C.J., et al., Preimplant Normothermic Liver Perfusion of a Suboptimal Liver Donated After Circulatory Death. Am J Transplant, 2016. 16(1): p. 353-7.

7. Mergental, H., et al., Transplantation of Declined Liver Allografts Following Normothermic Ex-Situ Evaluation. Am J Transplant, 2016. 16(11): p. 3235-3245.

8. Perera, T., et al., First human liver transplantation using a marginal allograft resuscitated by normothermic machine perfusion. Liver Transpl, 2016. 22(1): p. 120-4.

9. Ravikumar, R., et al., Liver transplantation after ex vivo normothermic machine preservation: a Phase 1 (first-in-man) clinical trial. Am J Transplant, 2016.

10. Brockmann, J., et al., Normothermic perfusion: a new paradigm for organ preservation. Ann Surg, 2009. 250(1): p. 1-6.

11. $\mathrm{Xu}, \mathrm{H}$., et al., Excorporeal normothermic machine perfusion resuscitates pig DCD livers with extended warm ischemia. J Surg Res, 2012. 173(2): p. e83-8.

12. Eisenbach, C., et al., An early increase in gamma glutamyltranspeptidase and low aspartate aminotransferase peak values are associated with superior outcomes after orthotopic liver transplantation. Transplant Proc, 2009. 41(5): p. 1727-30.

13. Olthoff, K.M., et al., Validation of a current definition of early allograft dysfunction in liver transplant recipients and analysis of risk factors. Liver Transpl, 2010. 16(8): p. 943-9.

14. NHS Blood and Transplant, Organ Donation and Transplantation Activity Report 2016/17. September 2017.

15. Angelico, R., et al., Normothermic Machine Perfusion of Deceased Donor Liver Grafts Is Associated With Improved Postreperfusion Hemodynamics. Transplant Direct, 2016. 2(9): p. e97.

16. Leithead, J.A., et al., Hepatic ischemia reperfusion injury is associated with acute kidney injury following donation after brain death liver transplantation. Transpl Int, 2013. 26(11): p. 1116-25.

17. Jay, C.L., et al., Ischemic cholangiopathy after controlled donation after cardiac death liver transplantation: a meta-analysis. Ann Surg, 2011. 253(2): p. 259-64.

18. Mourad, M.M., et al., Aetiology and risk factors of ischaemic cholangiopathy after liver transplantation. World J Gastroenterol, 2014. 20(20): p. 6159-69.

19. Reddy, S., et al., Non-heart-beating donor porcine livers: the adverse effect of cooling. Liver Transpl, 2005. 11(1): p. 35-8.

20. Abstracts of the 18th Congress of the European Society for Organ Transplantation, 24-27 September 2017, Barcelona, Spain. Transpl Int, 2017. 30 Suppl 2: p. 5-576.

\section{Figure Legends}

Figure 1: Image of liver during normothermic machine perfusion. The hepatic artery (HA), portal vein (PV), inferior vena cava (IVC) and common bile duct (CBD) are all cannulated. The gallbladder (GB) is also present although this was often removed during the retrieval process prior to NMP. This image has been used with donor family consent. 
Figure 2: CONSORT diagram. CONSORT diagram depicting the outcome for all donor livers enrolled in the trial. Key: ITT - Intention to treat PP - Per protocol

\section{Table 1: Donor demographic details}

\section{Table 2: Preservation and recipient demographic details.}

Key for tables 1 and 2: ^Median, IQR and full range reported. *Frequency and column percentages reported. \$ Functional warm ischaemia applies to DCD donors and is measured from the onset of functional warm ischaemia (systolic $\mathrm{BP}<50 \mathrm{mmHg}$ or $\mathrm{O} 2$ saturations $<70 \%$ ) to cross clamp. \#Measurement of the degree of steatosis was based on clinical assessment by the retrieval surgeon. $\ddagger C h i$-square and non-parametric Mann-Whitney $U$ test used for categorical and continuous variables, respectively. No adjustment for multiple comparisons were made

Table 3: Trial Outcomes. Key: \$Intention to treat (ITT) analysis adjusted for donor type and transplant centre. †Total number of livers transplanted and analysed overall. Primary outcome analysed on $\mathrm{N}=220$ due to unavailability of AST values in first 7 days post-transplant. Specific outcomes may have different denominators due to some missing data. ${ }^{*}$ Effect reported is: \% reduction (from geometric mean ratio) for Peak AST; odds ratio for EAD; difference in proportions (\%) for Discard rates, Post Reperfusion Syndrome and Need for RRT; not reported for outcomes where medians are reported, for survivals and for test for interaction for subgroup analysis (only $p$-value reported). \#Denominators for the discard rates is the total number of livers retrieved $(N=270, N M P=137, S C S=133)$. ${ }^{\wedge}$ Test not performed due to few events and no events in one arm. ¥Median and IQR reported, Mann-Whitney/ Wilcoxon rank-sum non-parametric test used.

\section{Acknowledgements:}

We would like to thank the European Commission for their generous support through the Seventh Framework Programme. The following organisations, groups and individuals also made substantial contributions without which this trial could not have been completed successfully: NHS Blood and Transplant; the Surgical Intervention Trials Unit, University of Oxford; the Clinical Trials and Research Governance unit, University of Oxford; Centre for Evidence in Transplantation, Royal College of Surgeons of England; the Liver Transplant Coordinators and liver unit physicians at the Queen Elizabeth Hospital, Birmingham, Addenbrooke's Hospital, Cambridge, King's College Hospital, London, and the Royal Free Hospital London; Dr Martin Soo, Dr Samuel Morrish, Mr Chris Morris, Ms Lucy Randle, Dr Rubens Macedo Arantes, Dr Reza Morovat, Dr Ahmed Elsharkawy, Prof Gideon Hirschfield, Dr John Isaac, Ms Jo Grayer, Dr Bettina Buchholz, Dr Hector Vilca-Melendez, Ms Ane Zamalloa, Dr Dimitrios Chasiotis, Dr Shirin Khorsandi, Prof Brian Davidson, Dr Dinesh Sharma, Ms Audrey Esson, Prof Diethard Monbaliu, Ms Sarah Mertens, Dr Sandra Swoboda, Dr Jan Neuhaus, Dr Tamas Benkö, Dr Victor Molina, Mr Rajeev Kumar, Ms Ally Bradley, Ms Margaux Laspeyres, Ms Alisanwe Mukwamba, Ms Sarah Banks, the Specialist Nurses in Organ Donation and, of course, all of the donors and their families.

\section{Author Contributions:}

DN, SK, CCC, and PJF designed this study with help from other authors. ZA and RJP oversaw the collection of samples and establishment of the trial biobank. DN, CC, AW, HM, MTP, DM, WJ, NH, Cl, MM, RR, AB, CW, IJ, JP, PK, AP, MP, JCGV were responsible for the clinical conduct of the study at the respective trial sites. DN, SK, VC and SD were responsible for statistical design and analysis. LR and CC provided device 
support and expertise to all trial sites. DN, SU, AP and JK were responsible for MRCP image analysis. PM, SK and SD provided governance oversight to ensure the study adhered to all regulatory and ethical requirements. All authors reviewed the manuscript.

\section{Methods}

\section{Study Design}

This investigator-led, multinational, open-label, two-arm, parallel randomised controlled trial included seven liver transplant centres from the UK (Addenbrooke's Hospital, Cambridge; King's College Hospital, London; Queen Elizabeth Hospital, Birmingham; Royal Free Hospital, London), Belgium (Universitaire Ziekenhuizen, Leuven), Spain (Hospital Clínic de Barcelona) and Germany (Universitatsklinikum, Essen), and was part of the EU funded Consortium for Organ Preservation in Europe (COPE, www.cope-eu.org). Approval was obtained from national research ethics committees and medical device regulatory bodies in each trial region, in particular the London-Dulwich National Research Ethics Committee (NREC) and the Medicines and Healthcare Regulatory Agency (MHRA) in the UK; the Federaal Agentschap voor Geneesmiddelen en Gezondheidsproducten (FAGG) and the Commissie Medische Ethiek of Universitaire ziekenhuizen, Leuven, Belgium; the Comité Ético de Investigación Clínica of the Hospital Clínic de Barcelona; the Deutsche Ärztekammer and the ethics committee of University Hospital Essen, Germany. The trial protocol was registered prior to recruitment (ISRCTN 39731134). All relevant ethical regulations relating to the conduct of this study were followed at each trial site. The trial is reported in accordance with the CONSORT statement [21].

No major amendments were made to the trial design after the start of recruitment.

\section{Eligibility and Consent}

Inclusion criteria for donors and recipients were deliberately broad to represent the full spectrum of clinical practice. Whole livers from DBD and DCD (Maastricht category III [22]) donors aged at least 16 years were eligible. Specific donor consent was not required for trial inclusion. No organs were procured from prisoners. Recipients were eligible provided they were at least 18 years old and listed for a liver-only transplant, excluding those with fulminant liver failure, due to the poor prognosis of this group regardless of organ quality. Potential participants were consented whilst on the waiting list; consent was affirmed on the day of transplant. The consent included the recording of anaonymised data for trial purposes and the collection of biological samples for storage in the trial biobank (see Sample Collection section below). No patient identifiable data was collected.

\section{Randomisation}

Once an eligible donor organ was allocated to a consented recipient and the availability of the NMP device and team was confirmed, the liver was randomised. All clinical decisions thereafter, including graft suitability and procedure scheduling, were made independently of the trial team.

Using an online randomisation tool, livers were assigned to NMP or SCS with 1:1 allocation ratio as per a computer generated randomisation schedule, using variable block size, stratified by transplant centre and donor type (DBD/DCD). The unit of randomisation was donor livers rather than recipients, but analysis is reported for the transplant recipients.

\section{Static Cold Storage group}

Livers randomised to SCS were retrieved, preserved, transported and transplanted according to local standard practice. 


\section{Normothermic Machine Perfusion group}

The OrganOx metra ${ }^{\circledR}$ normothermic liver perfusion device was used (Extended data Figure 5a), which enables automated organ preservation for up to 24 hours. Following randomisation to NMP, the device and accompanying researcher were transported to the donor hospital. The device was set-up during the retrieval procedure, as has been previously described [9]. A sterile disposable set was installed on to the device and primed with $500 \mathrm{ml}$ Gelofusine (B Braun Ltd) and three units of donor-matched packed red cells. Antibiotics were given at the outset and heparin, insulin, prostacyclin, bile salts and fat-free TPN were infused during the perfusion (Extended data figure $5 b$ ).

Following retrieval of the donor organ, and whilst still at the donor hospital, the liver back-table operation was performed [23], followed by cannulation of the hepatic artery, portal vein, inferior vena cava and bile duct. The liver was connected to the NMP device and perfusion commenced (Figure 1). During the early part of the perfusion sodium bicarbonate was added incrementally to achieve a physiological $\mathrm{pH}$. The OrganOx metra ${ }^{\circledR}$ perfusion device incorporates on-line blood gas measurement (Terumo CD1-500) together with software-controlled algorithms to control $\mathrm{PO}_{2} \& \mathrm{PCO}_{2}$ (within physiological limits), temperature $\left(37^{\circ} \mathrm{C}\right)$, mean arterial pressure $(65-75 \mathrm{~mm} \mathrm{Hg})$ and IVC pressure $(0-2 \mathrm{~mm} \mathrm{Hg})$. Typical blood flows of $200-400 \mathrm{ml} / \mathrm{min}$ (artery) and $1000-1200 \mathrm{ml} / \mathrm{min}$ (portal vein) were obtained. Glucose was measured manually and the value entered into the device. If glucose fell below $10 \mathrm{mmol} / \mathrm{L}$ this automatically triggered the infusion of a fat-free TPN mixture (Nutriflex Special, B Braun) into the perfusate.

NMP continued throughout the duration of transport and storage until the transplanting team were ready to implant the liver. The minimum protocol-stipulated NMP duration was 4 hours, the time needed for ATP repletion in animal studies [11]. The maximum allowed NMP duration was 24 hours in line with the experience in the phase I study and the regulatory approval for the device [9].

\section{Sample Collection}

Tissue biopsies (donor liver and bile duct), recipient blood and urine were collected at pre-specified time points from every liver/transplanted patient in the study. In addition to these, samples of perfusate fluid and bile were collected from every NMP liver. These were stored in a central biobank established by the COPE Consortium for use in on-going mechanistic studies. Each sample was allocated a unique bar code which the biobank coordinator was able to match to a specific trial ID. No patient identifiable data was associated with each sample.

\section{Study End Points}

The primary endpoint was defined as the difference between the two treatment arms in the peak level of serum aspartate transaminase (AST) within seven days post-transplant. This is a clinically accepted biomarker, predictive of primary non-function as well as graft and patient survival $[12,24]$ and also associated with histological evidence of moderate to severe perfusion injury $[25,26]$.

A surrogate marker of graft survival was used in this trial for two reasons: (i) the relatively high survival rates in liver transplantation ( $>90 \%$ ) and, (ii) the multifactorial causes of graft loss. A trial based directly on graft or patient survival would need to be unfeasibly large.

In order to ensure consistency and to minimise the hypothetical AST 'wash-out' effect in the NMP-treated organs, the first post-transplant value was measured between 12 and 24 hours post-reperfusion.

Secondary end-points included:

- Organ discard rate (after retrieval);

- Post-reperfusion syndrome [27]: $>30 \%$ drop in mean arterial pressure persisting for $>1$ minute within five minutes of reperfusion; 
- Primary non-function: irreversible graft dysfunction, for non-technical and non-immunological causes, leading to death or emergency liver replacement during the first 10 days after liver transplantation;

- Early allograft dysfunction [13]: any one of: (i) bilirubin $>170 \mu \mathrm{mol} / \mathrm{l}$ on day 7 post-transplant; (ii) INR >1.6 on day 7 post-transplant; (iii) peak-AST >2000 IU/L during the first 7 days;

- Length of hospital and ITU stays;

- Need for renal replacement therapy;

- Evidence of cholangiopathy on MRCP at six months;

- Graft and patient survival at one year.

Full details of all secondary outcomes are available in the trial protocol (DOI : 10.1038/protex.2018.027).

\section{Six Month MRCP}

An MRCP scan was performed six months (range 5 to 7) after transplant to evaluate the biliary tree for features of cholangiopathy evident by biliary strictures. All scans were reviewed by two independent, radiologists blinded to the method of organ preservation with disparities adjudicated by a third radiologist. Due to the lack of any existing grading system for biliary strictures, a system was agreed in advance by consensus among the radiologists to allow definitive categorisation of the presence and site of strictures. The findings were reported as follows: i) normal biliary tree; ii) anastomotic stricture (>70\% of luminal diameter); iii) unequivocal evidence of non-anastomotic stricture anywhere in the biliary tree; iv) both anastomotic and non-anastomotic biliary strictures.

\section{Statistical Analysis}

Previous data from Universitaetsklinikum Essen, Germany, (unpublished), demonstrated the geometric mean of peak AST to be $608.59 \mathrm{IU} / \mathrm{L}$ in patients transplanted following SCS. The present study was powered to detect a (clinically-relevant) $33 \%$ reduction in peak AST with $90 \%$ power at a $5 \%$ significance level, requiring 220 transplanted livers (110 per arm).

Results are reported as a modified intention-to-treat (ITT) analysis. A per-protocol sensitivity analysis was also performed excluding livers that received machine perfusion outside the protocol specified range (424 hours) and comparing the groups according to the treatment actually received. Livers randomized but not retrieved were excluded from the analysis.

Primary outcome was analysed using ANOVA with adjustment for stratification factors. The peak AST was calculated for each recipient with at least 2 values available. Missing AST values were not imputed. Binary outcomes were assessed using test for proportions or logistic regression to adjust for potential confounders and report odds ratios. Continuous outcomes were compared using the T-test if normally distributed, or by Mann-Whitney $U$ test. Time-to-event outcomes were analysed using Kaplan-Meier estimates and log-rank tests. Outcomes are reported with 95\% confidence intervals and p-values to 3 decimal places. A p-value less than 0.05 was regarded as statistically significant.

Pre-specified subgroup analyses were performed for donor type (DCD vs DBD), donor risk index (ET-DRI) and MELD score using test for interaction and reported using forest plots. Interaction methods were used to look for consistency of treatment effect across the different subgroups and reported using forest plots. The study was not powered to detect differences in the subgroups; these results should only be regarded as hypothesis-generating.

Analyses were conducted with the use of Stata version 14.2 (StataCorp, College Station, TX).

No formal interim analyses of end-points were carried out. At regular intervals, an independent Data Monitoring Committee reviewed confidential reports covering recruitment, safety parameters and primary endpoint data. 
Full details of the statistical methodology are available in the Supplementary Information: Statistical Analysis Plan.

\section{Machine Perfusion Parameters}

During NMP continuous displays of pressures, flows, metabolic $(\mathrm{pH})$ and synthetic (bile production) liver function were available to the operator. In addition, lactate measurements were carried out using external blood gas analysis. The trial protocol did not stipulate the manner in which these parameters should be interpreted.

Once trial recruitment was complete, an ad hoc analysis was performed in which NMP organs were categorised according to those which, following transplant, displayed minimal preservation injury (MPI; peak AST <250 IU/litre) and those with severe preservation injury (SPI; peak AST >1000 IU/litre). Groups were compared for differences in donor and recipient characteristics, perfusate biochemistry, bile production and evidence of post-reperfusion syndrome.

\section{Adverse Events}

Reporting of adverse events (AE) was in accordance with the European Commission MEDDEV guidelines [28]. Following trial completion, these were reviewed by two independent clinicians blinded to the treatment arm. AEs with a Clavien-Dindo [29] grading $\geq 1 \mathrm{llb}$ were considered serious adverse events (SAEs). Rates of AEs are reported with $95 \%$ confidence intervals. No statistical tests were applied to these data.

Full details of the trial methodology are available in the Clinical Trial Protocol at DOI: 10.1038/protex.2018.027.

21. Schulz, K.F., et al., CONSORT 2010 statement: updated guidelines for reporting parallel group randomised trials. BMJ, 2010. 340: p. c332.

22. Kootstra, G., J.H. Daemen, and A.P. Oomen, Categories of non-heart-beating donors. Transplant Proc, 1995. 27(5): p. 2893-4.

23. Makowka, L., et al., Surgical technique of orthotopic liver transplantation. Gastroenterol Clin North Am, 1988. 17(1): p. 33-51.

24. Glanemann, M., et al., Clinical implications of hepatic preservation injury after adult liver transplantation. Am J Transplant, 2003. 3(8): p. 1003-9.

25. Gaffey, M.J., et al., Predictive value of intraoperative biopsies and liver function tests for preservation injury in orthotopic liver transplantation. Hepatology, 1997. 25(1): p. 184-9.

26. Karayalcin, K., et al., The role of dynamic and morphological studies in the assessment of potential liver donors. Transplantation, 1994. 57(9): p. 1323-7.

27. Hilmi, I., et al., The impact of postreperfusion syndrome on short-term patient and liver allograft outcome in patients undergoing orthotopic liver transplantation. Liver Transpl, 2008. 14(4): p. 5048.

28. Commission, E., European Commission Guidelines on Medical Devices Clinical Investigations: Serious Adverse Event Reporting (MEDDEV 2.7/3). Dec 2010.

29. Dindo, D., N. Demartines, and P.A. Clavien, Classification of surgical complications: a new proposal with evaluation in a cohort of 6336 patients and results of a survey. Ann Surg, 2004. 240(2): p. 205-13. 


\section{Data Availability Statement}

The data that support the findings of this study are available from the corresponding author upon reasonable request. The full Trial Protocol, Statistical Analysis Plan and Final Statistical Report are available in the Supplementary Information.

\section{Extended Data Legends}

Figure 1: Forest plot for subgroup analysis of Peak AST by donor type. Geometric mean ratio and 95\% confidence interval reported for each subgroup and overall. DBD group - 87 NMP, 80 SCS; DCD group - 33 NMP, 20 SCS.

Figure 2a-b title: Post-reperfusion syndrome

Figure 2a: Kaplan-Meier plot for 1 year patient survival with two-sided log-rank test

Figure 2b: Kaplan-Meier plot for 1 year graft survival with two-sided log-rank test

Figure 3a-d title: Machine perfusion parameters during NMP

Figure 3a: Hepatic artery flow during NMP. Mean and SD shown at each time point. Actual values shown in table below. $\mathrm{N}=87$

Figure 3b: Portal vein flow during NMP. Mean and SD shown at each time point. Actual values shown in table below. $\mathrm{N}=87$

Figure 3c: Perfusate $\mathrm{pH}$ during NMP. Mean and SD shown at each time point. Actual values shown in table below. $\mathrm{N}=87$

Figure 3d: Bile production during NMP. Mean and SD shown at each time point. Actual values shown in table below. $\mathrm{N}=87$

Figure 4: Perfusate lactate levels during NMP. Scatter Graph with Trend Line Showing Perfusate Lactate Levels at Different Time Points during NMP for all Transplanted Livers. N=94

Figure 5a-b title: Normothermic machine perfusion device and circuit

Figure 5a: OrganOx metra ${ }^{\circledR}$ (generation 1). The normothermic machine perfusion device used in the trial

Figure 5b: OrganOx metra ${ }^{\circledR}$ normothermic machine perfusion circuit. The liver is perfused via the hepatic artery and portal vein. It drains via the inferior vena cava to a centrifugal pump from where perfusate passes, via a heat exchanger/oxygenator, either to a reservoir or directly into the hepatic artery. The perfusate in the reservoir drains under gravity into the portal vein.

Table 1: Detailed breakdown of reasons for discard of NMP livers

Table 2a-c title: Post-reperfusion syndrome analysis

Table 2a: Post-reperfusion syndrome by treatment group. Frequencies and column percentages reported. Difference in proportions was tested using Fisher test for proportions

Table 2b: Difference in post-reperfusion lactate in the recipient in each treatment arm. This relates to the first lactate measurement recorded by the anaesthetist after liver reperfusion and occurred within 30 minutes of reperfusion. Analysis using non-parametric Mann-Witney U test. Key: IQR inter-quartile range

Table 2c: Difference in use of vasopressor medications prior to, during, and following liver reperfusion in the recipient. Percentage of total events reported in brackets. Details of specific vasopressors used were not recorded

Table 3a-b title: Extended primary outcome analysis

Table 3a: Primary outcome results from the unadjusted analysis. Sample size for analysis of primary outcome is $\mathrm{N}=220$. T-test used is two-sided. Key: ${ }^{\wedge}$ First cell in this column refers to the mean difference in natural logarithm Peak AST (variable used to run the analysis models). The second cell in this column refers to the geometric mean ratio of the Peak AST, used to look at the reduction in the original measurement. 
Table 3b: Treatment effect on Peak AST for donor type subgroups. Sample size for subgroup analysis is $\mathrm{N}=220$ (DBD group - 87 NMP, 80 SCS; DCD group - 33 NMP, 20 SCS). Test used is two-sided. No power calculation or adjustment made for subgroup analysis.

Table 4a-b title: Characteristics and perfusate analysis of livers included in NMP liver quality model development

Table 4a: Demographic data for organ quality model development livers. Demographic data for minimal preservation injury (MPI; peak AST <250 IU/litre; $n=28$ ) and significant preservation injury (SPI; peak AST >1000 IU/litre; $n=25$ ) groups. Continuous variables were analysed using an unpaired student's t-test and categorical variables using Fisher's exact test. Data presented as mean \pm SEM

Table 4b: Comparison of NMP perfusate analyses between MPI (peak AST <250 IU/litre; n=28) and SPI (peak AST >1000 IU/litre; $n=25$ ) groups. D'Agostino Pearson normality test performed to assess data distribution. Parametric data were analysed using an unpaired student's t-test and non-parametric data were analysed using a Mann-Whitney $U$ test. Parametric data are presented as mean \pm SEM and non-parametric data are presented as median and range

Table 5a-c title: Adverse Events Analysis

Table 5a: Number of patients with any adverse events reported in each trial arm. Percentage of total events reported in brackets. No statistical tests have been applied.

Table 5b: Adverse events categorized by Clavien-Dindo grade. Breakdown of adverse events in each trial arm according to Clavien-Dindo grading. Percentage of total events reported in brackets. Adverse events with Clavien-Dindo grading $\geq \mathrm{IIIb}$ categorized as Serious Adverse Events. No statistical tests have been applied.

Table 5c: Breakdown of Adverse Events and Serious Adverse Events in each trial arm. Percentage of total events reported in brackets. Adverse events with Clavien-Dindo grading $\geq \mathrm{IIIb}$ categorized as Serious Adverse Events. No statistical tests have been applied.

Table 6: Detailed breakdown of adverse events in each trial arm. Percentage of total events reported in brackets. No statistical tests have been applied. 
Table 1

\begin{tabular}{|c|c|c|}
\hline Stratification factors (all randomised livers) & NMP (N = 170) & $\operatorname{SCS}(N=164)$ \\
\hline \multicolumn{3}{|l|}{ Donor type* } \\
\hline DBD & $107(62.9 \%)$ & $104(63.4 \%)$ \\
\hline DCD & $63(37.1 \%)$ & $60(36.6 \%)$ \\
\hline Donor demographics (retrieved livers) & NMP ( $N=137)$ & $\operatorname{SCS}(N=133)$ \\
\hline \multicolumn{3}{|l|}{ Gender* } \\
\hline Female & $54(39.4 \%)$ & $57(42.9 \%)$ \\
\hline Male & $81(59.1 \%)$ & $76(57.1 \%)$ \\
\hline (missing) & $2(1.5 \%)$ & $0(0.0 \%)$ \\
\hline Age $^{\wedge}$ & $56(45,67)(16,84)$ & $56(47,66)(20,86)$ \\
\hline \multicolumn{3}{|l|}{ Ethnicity* } \\
\hline African-Caribbean & $3(2.2 \%)$ & $1(0.8 \%)$ \\
\hline Caucasian & $131(95.6 \%)$ & $128(96.2 \%)$ \\
\hline Other & $1(0.7 \%)$ & $4(3.0 \%)$ \\
\hline (missing) & $2(1.5 \%)$ & $0(0.0 \%)$ \\
\hline \multicolumn{3}{|l|}{ Cause of death } \\
\hline CVA & $74(54.0 \%)$ & $74(55.6 \%)$ \\
\hline Hypoxia & $30(21.9 \%)$ & $32(24.1 \%)$ \\
\hline Trauma & $17(12.4 \%)$ & $16(12.0 \%)$ \\
\hline Other & $14(10.2 \%)$ & $11(8.3 \%)$ \\
\hline (missing) & $2(1.5 \%)$ & $0(0.0 \%)$ \\
\hline BMI^ & $\begin{array}{c}26.26(23.66,30.52) \\
(16.42,46.65)\end{array}$ & $\begin{array}{c}27.01(23.74,30.56) \\
(17.24,49.96)\end{array}$ \\
\hline (missing) & $2(1.5 \%)$ & $0(0.0 \%)$ \\
\hline ET-Donor risk index^ & $\begin{array}{c}1.72(1.47,2.09) \\
(0.98,4.31)\end{array}$ & $\begin{array}{c}1.72(1.50,2.10) \\
(1.06,3.49)\end{array}$ \\
\hline (missing) & $16(11.7 \%)$ & $19(14.3 \%)$ \\
\hline
\end{tabular}


Table 2

\begin{tabular}{|c|c|c|c|}
\hline Preservation Details (transplanted livers) & NMP ( $N=121)$ & $\operatorname{SCS}(N=101)$ & p-value $\ddagger$ \\
\hline $\begin{array}{l}\text { Functional warm ischaemia time } \$ \text { (minutes) } \\
\text { (Applies to DCD livers; } N=55,34 \text { NMP, } 21 \text { SCS) }\end{array}$ & $21(17,25)(9,93)$ & $16(10,20)(2,32)$ & 0.003 \\
\hline $\begin{array}{l}\text { Cold ischaemia time prior to NMP (minutes) } \\
(\mathrm{N}=120)\end{array}$ & $\begin{array}{c}126(106.5,143)(49, \\
218)\end{array}$ & - & \\
\hline $\begin{array}{l}\text { Machine perfusion time (minutes) } \\
(\mathrm{N}=120)\end{array}$ & $\begin{array}{l}547.5(372.5,710.5) \\
\quad(85,1388)\end{array}$ & - & \\
\hline $\begin{array}{l}\text { Total Preservation time from cross clamp in donor to } \\
\text { organ reperfusion in recipient (minutes) }\end{array}$ & $\begin{array}{c}714(542,876)(258 \\
1527)\end{array}$ & $\begin{array}{c}465(375,575)(223 \\
967)\end{array}$ & 0.0000 \\
\hline Steatosis assessed pre-preservation*\# & & & 0.366 \\
\hline None or mild & $91(75.3 \%)$ & $89(88.2 \%)$ & \\
\hline Moderate or severe & $29(24 \%)$ & $12(11.9 \%)$ & \\
\hline (missing) & $1(0.8 \%)$ & & \\
\hline Recipient demographics & NMP $(N=121)$ & $\operatorname{SCS}(N=101)$ & p-value $\ddagger$ \\
\hline Gender* & & & 0.717 \\
\hline Female & $35(28.9 \%)$ & $27(26.7 \%)$ & \\
\hline Male & $86(71.1 \%)$ & $74(73.3 \%)$ & \\
\hline Donor type* & & & 0.209 \\
\hline DBD & $87(71.9 \%)$ & $80(79.2 \%)$ & \\
\hline DCD & $34(28.1 \%)$ & $21(20.8 \%)$ & \\
\hline $\operatorname{Age}^{\wedge}$ & $55(48,62)(20,72)$ & $55(48,62)(22,70)$ & 0.713 \\
\hline Cause of Liver Failure* & & & 0.782 \\
\hline Alcoholic & $36(29.8 \%)$ & $29(28.7 \%)$ & \\
\hline Auto-Immune Hepatitis & $2(1.7 \%)$ & $5(5.0 \%)$ & \\
\hline Hepatitis B & $3(2.5 \%)$ & $2(2.0 \%)$ & \\
\hline Hepatitis C & $4(3.3 \%)$ & $4(4.0 \%)$ & \\
\hline Hepatocellular Carcinoma on background of Cirrhosis & $15(12.4 \%)$ & $16(15.8 \%)$ & \\
\hline Non-Alcoholic Steato-Hepatitis & $11(9.1 \%)$ & $11(10.9 \%)$ & \\
\hline Primary Biliary Cirrhosis & $10(8.3 \%)$ & $3(3.0 \%)$ & \\
\hline Primary Sclerosis Cholangitis & $18(14.9 \%)$ & $13(12.9 \%)$ & \\
\hline Other & $22(18.1 \%)$ & $18(17.8 \%)$ & \\
\hline $\mathrm{BMI}^{\wedge}$ & $\begin{array}{l}26.18(23.12,32.39) \\
\quad(18.02,50.99)\end{array}$ & $\begin{array}{l}26.94(24.36,30.42) \\
(18.91,42.95)\end{array}$ & 0.626 \\
\hline (missing) & $0(0.0 \%)$ & $1(1.0 \%)$ & \\
\hline Retransplant* & $12(9.9 \%)$ & $8(7.9 \%)$ & 0.605 \\
\hline MELD score^ (calculated at time of transplant) & $13(10,18)(6,35)$ & $14(9,18)(6,29)$ & 0.970 \\
\hline UK & $13(10,17)(6,33)$ & $14(9,18)(6,28)$ & \\
\hline Essen, Germany & $17(14,19)(13,23)$ & $15.5(14,17)(14,17)$ & \\
\hline Barcelona, Spain & $16(8,26)(8,35)$ & $14(9,16)(8,29)$ & \\
\hline Leuven, Belgium & $19(13.5,25)(13,26)$ & $16(16,20)(9,27)$ & \\
\hline eGFR^ & $\begin{array}{c}87.36(69.61,107.66) \\
(33.45,156.43)\end{array}$ & $\begin{array}{c}92.22(69.72,104.24) \\
(30.19,155.04)\end{array}$ & 0.928 \\
\hline (missing) & $4(3.3 \%)$ & $3(3.0 \%)$ & \\
\hline
\end{tabular}




\begin{tabular}{|c|c|c|c|}
\hline ET-Donor risk index^ & $1.70(1.47,2.07)$ & $1.71(1.50,2.01)$ & 0.610 \\
\hline (missing) & $(0.98,4.31)$ & $(1.063 .49)$ & $13(12.9 \%)$ \\
\hline
\end{tabular}

Table 3

\begin{tabular}{|c|c|c|c|c|}
\hline & $\begin{array}{c}\text { NMP } \\
(N=121)^{\dagger}\end{array}$ & $\begin{array}{c}\text { SCS } \\
(N=101)^{\dagger}\end{array}$ & Effect (95\% C.I.) & p-value \\
\hline \multicolumn{5}{|l|}{ Peak AST } \\
\hline \multicolumn{5}{|l|}{$\mathrm{ITT}^{\$}$} \\
\hline adjusted & $\begin{array}{c}488.1 \\
(408.9,582.8)\end{array}$ & $\begin{array}{c}964.9 \\
(794.5,1172.0)\end{array}$ & $0.5(0.4,0.7)$ & 0.0000 \\
\hline unadjusted & $\begin{array}{c}484.5 \\
(406.4,577.6)\end{array}$ & $\begin{array}{c}973.7 \\
(795.2,1192.3)\end{array}$ & $0.5(0.4,0.6)$ & 0.0000 \\
\hline $\begin{array}{l}\text { Test for interaction by donor } \\
\text { type }\end{array}$ & & & & 0.012 \\
\hline \multicolumn{5}{|l|}{$\begin{array}{l}\text { Subgroup analysis by donor } \\
\text { type }\end{array}$} \\
\hline DBD & $\begin{array}{c}526.2 \\
(427.3,647.9)\end{array}$ & $\begin{array}{c}880.2 \\
(708.5,1093.5)\end{array}$ & $\begin{array}{l}40.2 \%(19.3 \% \\
55.7 \%)\end{array}$ & 0.0009 \\
\hline $\mathrm{DCD}$ & $\begin{array}{c}389.7 \\
(278.0,546.4)\end{array}$ & $\begin{array}{c}1458.1 \\
(944.7,2250.5)\end{array}$ & $\begin{array}{c}73.3 \%(53.7 \% \text { to } \\
84.6 \%,)\end{array}$ & 0.0000 \\
\hline PP analysis & $\begin{array}{c}498.6 \\
(414.8,599.4)\end{array}$ & $\begin{array}{c}982.9 \\
(810.4,1192.2)\end{array}$ & $0.5(0.4,0.7)$ & 0.0000 \\
\hline Discard rates $\#$ & $16(11.7 \%)$ & $32(24.1 \%)$ & $\begin{array}{c}-12.4 \%(-21.4 \%,- \\
3.3 \%)\end{array}$ & 0.008 \\
\hline Primary non-function ${ }^{\wedge}$ & $1(0.8 \%)$ & $0(0.0 \%)$ & - & - \\
\hline Post reperfusion syndrome & $15(12.4 \%)$ & $32(33.0 \%)$ & $\begin{array}{c}-20.6 \%(-31.6 \%,- \\
9.6 \%)\end{array}$ & 0.0002 \\
\hline Post reperfusion lactate $e^{\ddagger}$ & $3.6(2.6,4.2)$ & $4.1(3.2,5)$ & & 0.018 \\
\hline Early allograft dysfunction & $12(10.1 \%)$ & $29(29.9 \%)$ & $0.263(0.126,0.550)$ & 0.0002 \\
\hline \multicolumn{5}{|l|}{$\begin{array}{l}\text { Biochemical liver tests }^{\ddagger} \\
\text { (average value over Day 1-7) }\end{array}$} \\
\hline \multicolumn{5}{|l|}{ Bilirubin ( $\mu \mathrm{mol} / \mathrm{L})$} \\
\hline Day 1-7 & $38.5(21.0,73.2)$ & $49.1(26.0,85.5)$ & & 0.029 \\
\hline 30 days & $13.0(8.0,22.1)$ & $13.0(9.1,21.0)$ & & 0.479 \\
\hline 6 months & $9.1(6.0,15.1)$ & $9.1(6.0,13.0)$ & & 0.671 \\
\hline \multicolumn{5}{|l|}{ AST (IU/L) } \\
\hline Day 1-7 & $167.5(98,320.7)$ & $318.5(152,611.5)$ & & 0.0000 \\
\hline 30 days & $20(14,35)$ & $22(15,40)$ & & 0.707 \\
\hline 6 months & $23(18,33)$ & $23(18,37)$ & & 0.931 \\
\hline \multicolumn{5}{|l|}{ VGT (IU/L) } \\
\hline Day 1-7 & $268.1(156.3,408.3)$ & 301 (201.1, 443.9) & & 0.157 \\
\hline 30 days & $178(109.5,410)$ & $200(96,397.5)$ & & 0.949 \\
\hline 6 months & $47(28,144)$ & $47(26,128)$ & & 0.452 \\
\hline \multicolumn{5}{|l|}{ INR } \\
\hline Day 1-7 & $1.2(1.2,1.4)$ & $1.2(1.2,1.4)$ & & 0.644 \\
\hline 30 days & $1.1(1,1.2)$ & $1.1(1,1.2)$ & & 0.735 \\
\hline 6 months & $1.1(1,1.2)$ & $1.1(1,1.1)$ & & 0.167 \\
\hline \multicolumn{5}{|l|}{ Creatinine ( $\mu \mathrm{mol} / \mathrm{L})$} \\
\hline Day 1-7 & $92.8(60.1,121.1)$ & $97.2(67.2,143.2)$ & & 0.139 \\
\hline
\end{tabular}




\begin{tabular}{|c|c|c|c|c|}
\hline 30 days & $82.2(66.3,104.3)$ & $90.2(72.5,121.1)$ & & 0.019 \\
\hline 6 months & $99.9(81.3,117.6)$ & $99.9(83.1,134.4)$ & & 0.265 \\
\hline \multicolumn{5}{|l|}{ Lactate $(\mathrm{mmol} / \mathrm{L})$} \\
\hline Day 1-7 & $1.3(1,1.7)$ & $1.1(0.9,1.6)$ & \multicolumn{2}{|r|}{0.130} \\
\hline \multicolumn{5}{|l|}{$\begin{array}{l}\text { Need for RRT (No and \% of } \\
\text { patients) }\end{array}$} \\
\hline Day 1-7 post-transplant & $26(21.5 \%)$ & $19(18.8 \%)$ & $2.7 \%(-7.9 \%, 13.2 \%)$ & 0.621 \\
\hline Day 30 & $27(22.3 \%)$ & $20(19.8 \%)$ & $2.5(-8.2 \%, 13.3 \%)$ & 0.648 \\
\hline Month 6 & $27(22.3 \%)$ & $21(20.8 \%)$ & $1.5 \%(-9.3 \%, 12.4 \%)$ & 0.784 \\
\hline Duration of RRT Day 1-7 & $4(2,6)$ & $5(4,6)$ & & 0.346 \\
\hline Length of hospital stay ${ }^{\ddagger}$ & $15(10,24)$ & $15(11,24)$ & & 0.926 \\
\hline Length of HDU/ITU stay ${ }^{\ddagger}$ & $4(2,7)$ & $4(3,7)$ & & 0.339 \\
\hline Graft survival at 1 year & $0.950(0.893,0.977)$ & $0.960(0.897,0.985)$ & & 0.707 \\
\hline Patient survival at 1 year & $0.958(0.902,0.982)$ & $0.970(0.909,0.990)$ & & 0.671 \\
\hline
\end{tabular}

